\title{
Universiteit
}

Leiden

The Netherlands

\section{How boron nitride forms a regular nanomesh on $\mathbf{R h}(111)$}

Dong, G.; Fourré, E.B.; Tabak, F.C.; Frenken, J.W.M.

\section{Citation}

Dong, G., Fourré, E. B., Tabak, F. C., \& Frenken, J. W. M. (2010). How boron nitride forms a regular nanomesh on $\mathrm{Rh}(111)$. Physical Review Letters, 104(3), 096102.

doi:10.1103/PhysRevLett.104.096102

Version: $\quad$ Not Applicable (or Unknown)

License: $\quad$ Leiden University Non-exclusive license

Downloaded from: https://hdl.handle.net/1887/61247

Note: To cite this publication please use the final published version (if applicable). 


\title{
How Boron Nitride Forms a Regular Nanomesh on $\operatorname{Rh}(111)$
}

\author{
Guocai Dong, Elodie B. Fourré, ${ }^{*}$ Femke C. Tabak, and Joost W. M. Frenken ${ }^{\dagger}$ \\ Kamerlingh Onnes Laboratory, Leiden University, PO Box 9504, 2300 RA Leiden, The Netherlands
}

(Received 18 November 2009; published 3 March 2010)

\begin{abstract}
Boron nitride forms nearly perfectly regular films with a thickness of precisely one atom on various metal surfaces. Here, we follow the formation of boron nitride layers on $\mathrm{Rh}(111)$ with scanning tunneling microscopy (STM) under realistic growth conditions, up to $1200 \mathrm{~K}$. Our STM movies demonstrate in detail how the structure grows and how defects are introduced. Based on these observations we arrive at the optimal recipe for a high-quality overlayer.
\end{abstract}

DOI: 10.1103/PhysRevLett.104.096102

PACS numbers: 68.55.Ln, 68.37.Ef, 81.15.Gh

On the $\mathrm{Rh}(111)$ surface, hexagonal boron nitride ( $h$-BN) adopts a highly regular superstructure with $2 \mathrm{~nm}$ diameter depressions and a $3.2 \mathrm{~nm}$ period. Similar structures are found on other metal surfaces. This so-called nanomesh [1] serves as a two-dimensional scaffold for deposition of buckyballs and other, functional molecules [1,2], which may lead to interesting, e.g., electronic, magnetic or catalytic properties. The nanomesh coating has been demonstrated to remain intact and protect the underlying metal under ambient conditions [3], in liquids [4] and at high temperatures [1]. The atomic structure of the nanomesh has been unraveled by a combination of scanning tunneling microscopy (STM) [1,5], surface x-ray diffraction [3] and DFT calculations [6,7] to be a single, highly corrugated layer of $h$-BN, with $13 \times 13$ unit cells of the $h$-BN lattice fitting on $12 \times 12$ unit cells of the underlying $\mathrm{Rh}(111)$.

The central question in this Letter is: How can the overlayer with its large supercell of 338 overlayer atoms develop into a nearly perfect structure? The original procedure of Corso et al. for nanomesh growth is to expose $\mathrm{Rh}(111)$ to borazine $(\mathrm{HBNH})_{3}$ gas at $1050 \mathrm{~K}$ [1]. This high temperature could be necessary to crack the borazine molecules, releasing hydrogen and possibly rupturing $\mathrm{B}-\mathrm{N}$ bonds, in order to form reactive precursors that stick sufficiently strongly to the surface and serve as the growth units from which the overlayer can be assembled. One can also imagine that a high temperature would be required to provide sufficient lateral mobility to crystallize the otherwise disordered overlayer into the perfectly periodic nanomesh structure. While the high temperature serves as an important clue, it also poses a significant experimental difficulty.

We address the assembly of $h$-BN nanomesh films on $\mathrm{Rh}(111)$ by in situ STM observations under the reported growth conditions, i.e., during borazine deposition at temperatures up to $1200 \mathrm{~K}$. All measurements were carried out in ultrahigh vacuum with a variable-temperature STM that allows fast scanning and imaging over a wide range of temperatures and during substantial temperature changes $[8,9]$. The Rh sample was cleaned by a standard procedure
[10] and its quality was checked with STM and auger electron spectroscopy.

We started our investigation by exposing clean $\mathrm{Rh}(111)$ to $40 \mathrm{~L}$ (1 langmuir $=10^{-6}$ torr s) of borazine gas at room temperature. The STM images show that this was enough to saturate the Rh surface with a disordered deposit [Fig. 1(a)]. This clearly indicates that the high temperature
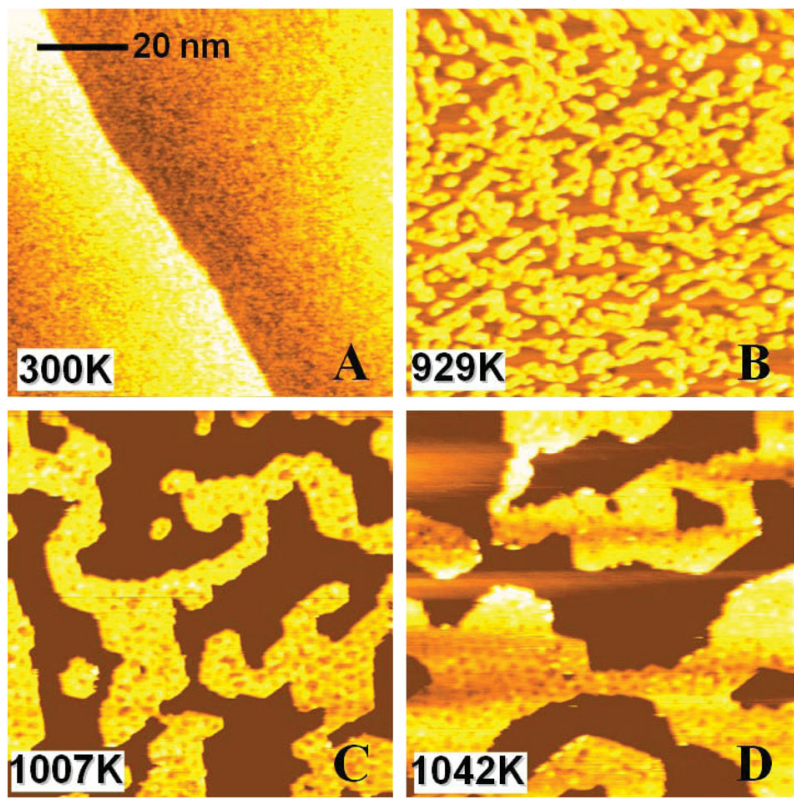

FIG. 1 (color). Series of images from an STM movie [12] during a continuous ramp from 300 to $1057 \mathrm{~K}$. (a) The $\mathrm{Rh}(111)$ surface directly after exposure to $40 \mathrm{~L}$ of borazine gas at room temperature. A monoatomic step on the Rh surface crosses the image. The rough appearance of the upper and lower terraces is caused by the borazine deposit. (b) At higher temperatures, the overlayer first organized into narrow, elongated $h$ - BN islands. The height variations within the islands result from the mismatch between the lattice constants of the overlayer and the substrate. (c) The next stage was the formation of more compact $h$-BN islands with a defective nanomesh superstructure. (D) At the final temperature the nanomesh structure was relatively well defined. Image size $85 \mathrm{~nm} \times 85 \mathrm{~nm}$; sample voltage $V_{b}=$ $3.0 \mathrm{~V}$; tunneling current $I_{t}=0.05 \mathrm{nA}$. 
of the nanomesh recipe is not necessary for $\mathrm{BN}$ to adsorb on the $\mathrm{Rh}$ surface.

In order to follow the formation of $h$-BN and its nanomesh superstructure directly, we heated up the preexposed $\mathrm{Rh}(111)$ surface slowly from room temperature to $\sim 1060 \mathrm{~K}$ while STM images were taken continually (Fig. 1). At $690 \mathrm{~K}$ we observed the first noticeable rearrangements into small, nanometer-scale clusters. As temperature increased further, these small clusters became mobile and coalesced. The branched and elongated shapes observed in Fig. 1(b) are characteristic of this diffusionlimited aggregation [11]. The distinct $0.05 \mathrm{~nm}$ height variations within the clusters [Fig. 1(b)] indicate that they already have the single-monolayer $h$-BN structure, with the corrugation resulting from the mismatch with the underlying Rh lattice. In Fig. 1(c) we see that at $1007 \mathrm{~K}$ the island shapes had become more compact and the height variations had organized into the nanomesh superstructure, albeit with a high density of defects. When the temperature was increased to $1042 \mathrm{~K}$, most defects disappeared and the islands ripened to larger sizes [Fig. 1(d)]. The images in Figs. 1(b)-1(d) show that the coverage was well below $100 \%$, even though the initial exposure of $40 \mathrm{~L}$ had been sufficient to reach maximum coverage at room temperature. However, our movies in the supplementary material [12] suggest no loss of material (borazine or $\mathrm{BN}$ ) during the heating, implying that almost all $\mathrm{B}$ and $\mathrm{N}$ atoms deposited at room temperature were still present at 1042 K. Two potential causes for the lack of material are (i) that at room temperature the borazine molecules formed a highly disordered and therefore noncompact layer [Fig. 1(a)], and (ii) that with most of their hydrogen atoms still present, the borazine molecules initially present at room temperature required more space than the final $h$-BN structure.

From this first experiment we have learned that the typical deposition temperature of $1050 \mathrm{~K}$ is not necessary for borazine adsorption or $h$-BN formation. Furthermore, we observed that the overlayer formed via twodimensional nucleation and growth. Because of the procedure adopted in this experiment, where we deposited the borazine at room temperature and subsequently heated the film, the resulting structure was far from a perfectly regular nanomesh. In order to find out what really limits the quality of growing nanomesh films it was necessary to perform STM observations under the reported optimal nanomesh formation conditions. Our STM observations show that also at $1050 \mathrm{~K}$ the overlayer formed via two-dimensional nucleation and growth, in this case with compact and wellseparated $h$-BN islands that immediately adopted a regular nanomesh structure. However, under these conditions, the average density of islands was so low, e.g., 3 islands $/ \mu \mathrm{m}^{2}$, that it was difficult to capture the interesting stages of the process. Therefore, we artificially increased the nucleation density to about 250 islands $/ \mu \mathrm{m}^{2}$ by a seeding procedure, which consisted of first exposing the Rh surface at room temperature to a low dose of $0.2 \mathrm{~L}$ borazine, followed by heating to $978 \mathrm{~K}$ [Fig. 2(a)]. On this seeded surface, we deposited further BN at $978 \mathrm{~K}$, while scanning continually by STM.

Figures 2(b)-2(d) are three snapshots from an STM movie [12] recorded during the growth of an $h$-BN nanomesh island at $978 \mathrm{~K}$. The initial, equilibrium island shape [Fig. 2(a)] was a hexagon with three short and three long edges, corresponding to a higher-energy and a lowerenergy termination of the island. When borazine was deposited, the island rapidly evolved into a (truncated) triangular growth shape [Fig. 2(b)]. In the movie and in Fig. 2 the growth appeared to proceed by the addition of entire nanomesh units. Although this may seem natural at first sight, it is surprising since each unit contains $169 \mathrm{~B}$ atoms and $169 \mathrm{~N}$ atoms. Careful inspection of the images revealed the presence of a highly mobile species at the island edges, which we interpret as $\mathrm{BN}$ diffusing along the island perimeter, until it locally accumulated into a new, complete nanomesh unit cell, at which point the atoms in this nano-

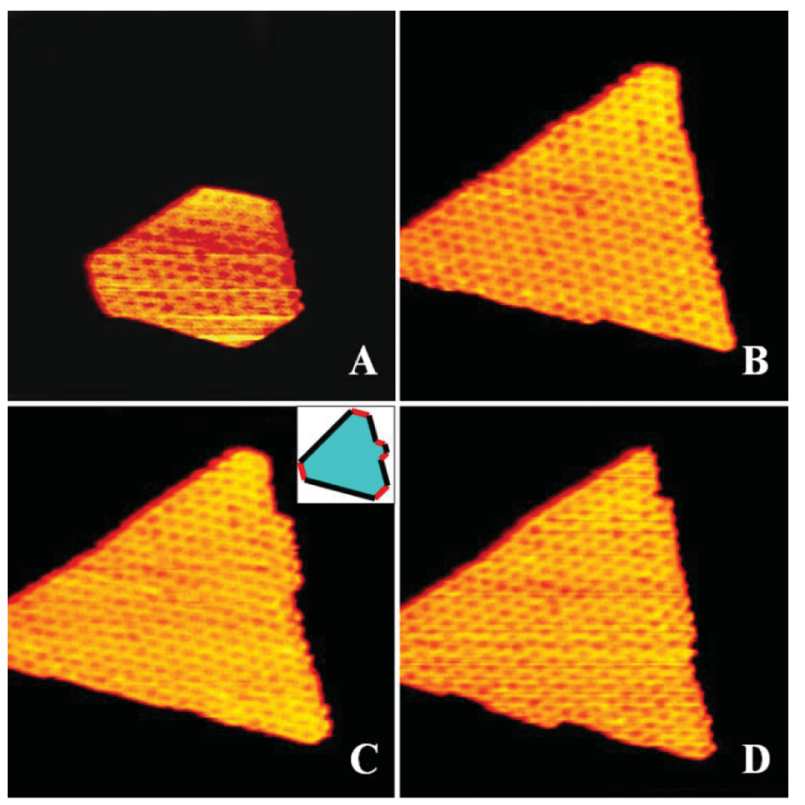

FIG. 2 (color). Images from an STM movie [12] of an $h$-BN island on $\mathrm{Rh}(111)$ during borazine deposition at $978 \mathrm{~K}$. (a) The initial island, obtained by $0.2 \mathrm{~L}$ borazine exposure at room temperature and subsequent annealing at $978 \mathrm{~K}$. (b) After $368 \mathrm{~s}$ of borazine exposure at $2.5 \times 10^{-9} \mathrm{mbar}$ at $978 \mathrm{~K}$; the island had become triangular within the first $60 \mathrm{~s}$. (c) After $421 \mathrm{~s}$ new kinks had formed on the right edge, while the kink at the bottom edge had advanced to the right corner. The inset is a sketch indicating the two edge types, the red one having the higher energy. (d) After $473 \mathrm{~s}$ the two kinks on the right edge had advanced and new kinks had formed on the lower edge. Image size $65 \mathrm{~nm} \times 65 \mathrm{~nm} ; V_{b}=1.0 \mathrm{~V} ; I_{t}=0.05 \mathrm{nA} ; 52.5 \mathrm{~s}$ per image. 
mesh unit were effectively immobilized in their new structure. We find that new nanomesh units were naturally forming at kinks, which made these kinks advance along the island perimeter (Fig. 2). Kinks were found to advance equally fast along the two edge types, the rate at which new kinks formed was lower along the lower-energy (longer) edges than along the high-energy (shorter) edges. This is why the growth shape deviates even further from an equalsided hexagon than the equilibrium shape from which the growth started. The lower kink creation rate on the lowerenergy edge is easy to understand. The size of a complete nanomesh unit cell is so large, that the energy of a kink along a low-energy edge will be relatively close to the energy of one unit cell of high-energy edge. Similarly, a kink along a high-energy edge will introduce an extra energy close to that for one unit cell of low-energy edge. The corresponding difference in kink energy will naturally make the kink creation rate lower on the low-energy edge. Since on both edge types a kink has the same local structure, connecting a high-energy and a low-energy segment, we should expect the $\mathrm{BN}$ deposition to make existing kinks move equally fast along the two types of edges, in accordance with our observations.

The observation of two different types of island edges implies that B-N bonds are broken at the growth temperature. Figure 3 shows that even though it is technically possible to construct a layer of $h$-BN without breaking $\mathrm{B}-\mathrm{N}$ bonds in the deposited borazine molecules, the edges of the resulting islands would all be equivalent, terminated by both $\mathrm{B}$ and $\mathrm{N}$ atoms (outer contour of the island in Fig. 3). Different edges, terminated exclusively by either $\mathrm{B}$ atoms or $\mathrm{N}$ atoms, can only be obtained by interrupting the layer along another direction (red line in Fig. 3) [13]. This edge direction is further supported by the observed orientation of the nanomesh pattern in the islands, as can be

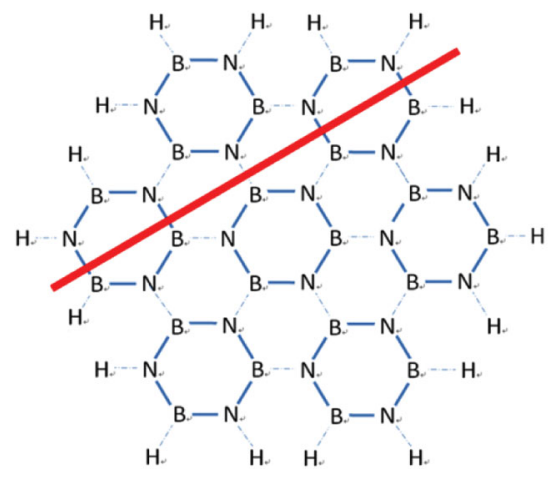

FIG. 3 (color). An $h$-BN overlayer can be formed without breaking $\mathrm{B}-\mathrm{N}$ bonds, by combining the intact $(\mathrm{BN})_{3}$ rings originating from borazine molecules. Blue solid lines denote bonds in the original molecules; the dashed ones are new and have formed in combination with the loss of hydrogen. The red solid line shows that only by cutting B-N bonds the island can develop two different types of edges, as seen in the STM images (e.g., Fig. 2), either $\mathrm{N}$ or B terminated. concluded from a comparison with atomically resolved images of the nanomesh structure and supporting theory in Refs. [5,6]. The breaking of B-N bonds forms a necessary step in the process, which probably requires a high temperature, as is suggested by the aggregates observed in Fig. 1(b) that only started rearranging into compact shapes around $930 \mathrm{~K}$.

In the final stage of the growth, the islands encountered each other and deposition stopped when the entire surface was covered. We found all locations where two islands merged to remain visible afterwards as defect lines in the nanomesh pattern [Figs. 4(a)-4(c)] [12]. The reason is that the probability for any two islands to match, even when they have precisely the same orientation, is as low as $1 / 144$. This is because each nanomesh unit cell covers $12 \times 12$ unit cells of the underlying $\mathrm{Rh}(111)$ surface, leading to 144 translational domains. A more detailed inspection showed that most defect lines appeared as a row of elongated, compressed and/or skewed nanomesh rings [Fig. 4(d)]. This probably means that in most cases the islands attached to each other by forming a fully continuous, distorted $h$-BN network, without any dangling bond. Like in any Moire pattern, the precise nanomesh period is very sensitive to small lattice distortions, a $4 \%$ local stretching already sufficing to double the size of a nanomesh unit. This effect makes the nanomesh pattern a

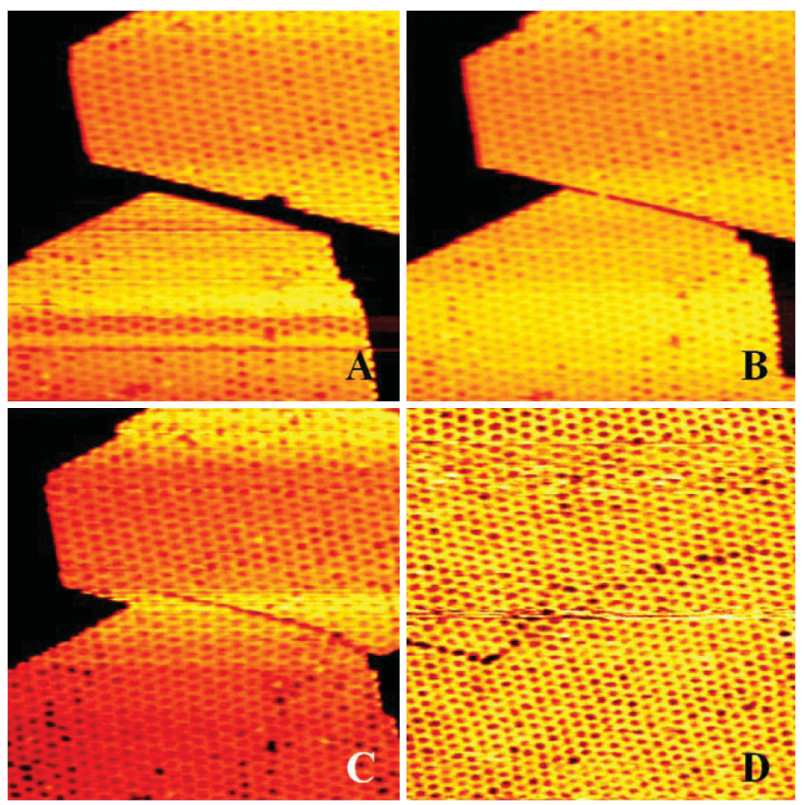

FIG. 4 (color). (a)-(c) Three subsequent images from an STM movie [12] showing the formation of a defect line in the $h$-BN nanomesh overlayer during growth in $1.2 \times 10^{-9}$ mbar borazine at $978 \mathrm{~K}$. The two merging islands share the same orientation but have a translational mismatch that remains visible after coalescence as a row of deformed nanomesh unit cells. These lines were surprisingly immobile, as is illustrated in (d), which was taken after the deposition had been completed and the fully $h$-BN covered Rh surface was heated further to $1135 \mathrm{~K}$. 
highly efficient magnifying glass for modest variations in the lattice constant. In addition to the translational phase differences, we also found $180^{\circ}$ misoriented islands, as had been found also for $h-\mathrm{BN}$ on Ni [13]. These introduced strong defect lines, probably in the $h$-BN lattice itself, when encountering properly oriented islands. This was to be expected, since a continuous network would otherwise have required the formation of N-N bonds or B-B bonds.

After the surface had been covered fully by $h$-BN, we heated the $\mathrm{Rh}$ crystal slowly to $1180 \mathrm{~K}$ in an attempt to remove the defects. To our surprise, all defect lines remained unchanged and static [Fig. 4(d)] until the $h$-BN layer started to disappear (desorb or dissolve) at $1160 \mathrm{~K}$. Note that we had observed the spontaneous removal of defect lines at lower temperatures [Figs. 1(c) and 1(d)], but these were much shorter, typically no more than one mesh unit. The $0.33 \mathrm{eV}$ bonding energy between $h-\mathrm{BN}$ and $\mathrm{Rh}$ [6] must make the activation energy for moving a longer defect line prohibitively large. We conclude that the structural quality of a nanomesh film cannot be improved by postdeposition treatments. The defect lines are a permanent fossil of the initial configuration of nuclei.

Combining all observations, we understand why the temperature needs to be as high as, e.g., $1050 \mathrm{~K}$ for the formation of a high-quality nanomesh film. Borazine is adsorbed readily at room temperature. The $h$-BN structure already emerges below $900 \mathrm{~K}$. B-N bonds can decompose already around $930 \mathrm{~K}$, allowing the overlayer to become compact. However, almost all places where nanomesh patches meet that originate from separate nuclei, result in line defects that cannot be removed. The only way to improve the quality of the film is therefore to minimize the density of such nuclei. Nucleation-and-growth theory predicts that the nucleation density should be a function of the ratio between the flux $F$ of deposited borazine molecules and the surface diffusion coefficient $D$ of these molecules (or their fragments) [14]. The best recipe is therefore to combine a low flux and a high diffusion coefficient. It is for the latter reason (high $D$ ) that a high temperature is a prerequisite. When the diffusion length has become so high that the nucleation is dominated by defects in the Rh substrate, a further increase in temperature will not significantly improve the film quality. Possibly, this limiting situation is typically reached at $1050 \mathrm{~K}$ for $h$-BN nanomesh films on $\mathrm{Rh}(111)$.

In view of the similarities between $h$-BN and graphene [15-19] and the generic nature of the behavior observed here, we expect that similar mechanisms are at play and similar recipes apply during the formation of boron nitride and graphene monolayers on a variety of transition-metal surfaces [19-22], for example, graphene on $\mathrm{Ni}(111)$ [15,23]. Preliminary, high-temperature STM observations of graphene growth on $\mathrm{Rh}(111)$ confirm this expectation.

The authors are indebted to Jürg Osterwalder and Thomas Greber (University of Zurich) for introducing them to the subject and for their help and to Hermann Sachdev (University of Saarland) for providing highquality borazine. This work was supported by the EU (No. FP6-013817).

*Present address: Ecole supérieure d'ingénieurs de Poitiers, Laboratoire de catalyse en chimie organique, University of Poitiers, 40, Avenue du Recteur Pineau, 86022 Poitiers Cedex, France.

frenken@physics.leidenuniv.nl

[1] M. Corso et al., Science 303, 217 (2004).

[2] H. Dil et al., Science 319, 1824 (2008).

[3] O. Bunk et al., Surf. Sci. 601, L7 (2007).

[4] R. Widmer et al., Electrochem. Comm. 9, 2484 (2007).

[5] S. Berner et al., Angew. Chem., Int. Ed. 46, 5115 (2007).

[6] R. Laskowski et al., Phys. Rev. Lett. 98, 106802 (2007).

[7] R. Laskowski and P. Blaha, J. Phys. Condens. Matter 20, 064207 (2008).

[8] M. S. Hoogeman et al., Rev. Sci. Instrum. 69, 2072 (1998).

[9] M. J. Rost et al., Rev. Sci. Instrum. 76, 053710 (2005).

[10] L. A. Delouise and N. Winograd, Surf. Sci. 138, 417 (1984).

[11] Z. Y. Zhang and M. G. Lagally, Science 276, 377 (1997).

[12] See supplementary material at http://link.aps.org/ supplemental/10.1103/PhysRevLett.104.096102 or http:// www.InterfacePhysics.nl for movies.

[13] W. Auwarter et al., Surf. Sci. 545, L735 (2003).

[14] J.A. Venables, Introduction to Surface and Thin Film Processes (Cambridge University Press, Cambridge, England, 2001).

[15] C. Oshima and A. Nagashima, J. Phys. Condens. Matter 9, 1 (1997).

[16] T. Kawasaki et al., Surf. Rev. Lett. 9, 1459 (2002).

[17] T. Tanaka et al., Surf. Rev. Lett. 10, 697 (2003).

[18] X. F. Gao et al., J. Phys. Chem. C 112, 12677 (2008).

[19] D. Martoccia et al., Phys. Rev. Lett. 101, 126102 (2008).

[20] A. Goriachko et al., Langmuir 23, 2928 (2007).

[21] A. B. Preobrajenski et al., Phys. Rev. B 78, 073401 (2008).

[22] B. Wang et al., Phys. Chem. Chem. Phys. 10, 3530 (2008).

[23] K. S. Kim et al., Nature (London) 457, 706 (2009). 